\title{
Erratum: First-principles study of pyroelectricity in GaN and $\mathrm{ZnO}$ [Phys. Rev. B 93, 081205(R) (2016)]
}

\author{
Jian Liu, P. B. Allen, and M. Fernández-Serra
}

(Received 11 July 2018; published 27 July 2018)

DOI: 10.1103/PhysRevB.98.039905

There was an error in Fig. 3 of this paper. The experimental temperature-dependent lattice parameters [1] $a(T)$ and $c(T)$ for $\mathrm{ZnO}$ were mistranscribed. This caused a significant error in the curve of $\Delta u_{\text {ext }}$. The error was not made when the derivative was plotted in Fig. 2. The results in Fig. 2 are correct, and the corrected version of Fig. 3 is given here. We also take this opportunity to add the predicted $\Delta u(T)$ for GaN, which was omitted from the original paper (the derivatives are shown in Fig. 1 of the original paper). We also wish to clarify some notations which were only implicit via the text but not included explicitly in the equations. The clarified version of Eq. (5) is

$$
p_{\epsilon}^{\beta}(T)=\frac{e}{\Omega} \sum_{j, \kappa \alpha} Z_{\epsilon}^{\beta \alpha}\left(\frac{\partial u_{\kappa \alpha}(\overrightarrow{0} j)}{\partial T}\right)_{\epsilon},
$$

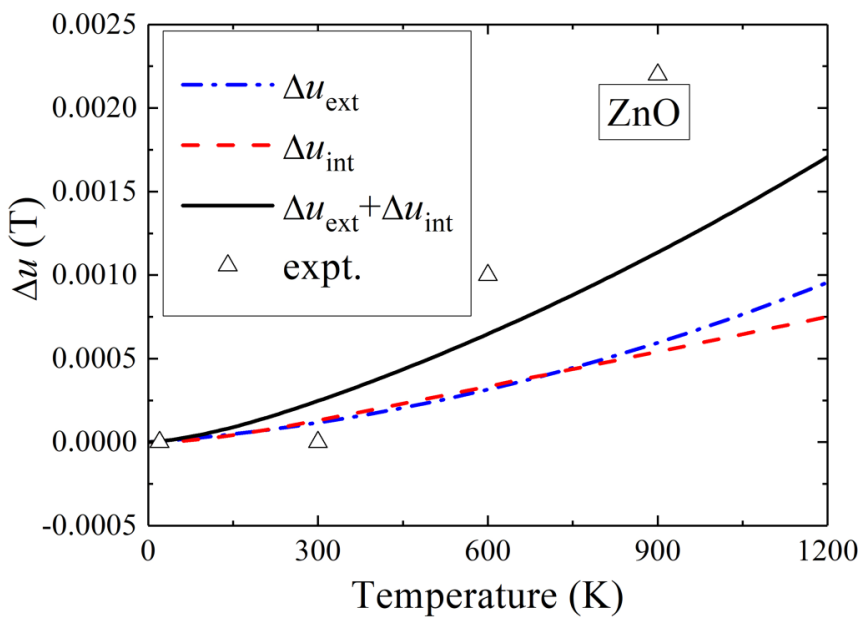

FIG. 1. Corrected version of Fig. 3: Thermal shift of the $\mathrm{ZnO}$ internal parameter $u$. The measured zero temperature value is 0.382 $[2,3]$, close to the "ideal" value of $3 / 8$. Experimental values are from Ref. [4]. The notation $\Delta u_{\text {int }}$ refers to the computed value at constant external strain $\epsilon$ [Eq. (7)], and $\Delta u_{\text {ext }}$ refers to the external straininduced effect [last two terms in Eq. (8)]. and the wurtzite version, two lines below in the text, should read $p_{\epsilon}(T)=(2 e / \Omega) Z^{*} c(\partial u / \partial T)_{\epsilon}$. Equation (7) is written for $\Delta u / u$, but, as the text explains, it is really $(\Delta u)_{\epsilon} / u$. The Grüneisen parameter $\gamma_{\vec{q} j}(0)$ used in that equation is identified correctly in the text as the "internal" Grüneisen parameter, defined in Eq. (A14). The second term in Eq. (8) is the internal shift [it should have been written as $\Delta u_{\text {int }}(T)-\Delta u_{\text {int }}(0)$ ], and the last two terms are the external shift [caused by external strains $\epsilon(T)$ ]. Finally, recent work has clarified and extended some of this paper. A more thorough discussion on the relation between external and internal thermal expansions can be found in Ref. [5]. New measurements of $p(T)$ are now available for GaN [6]. The electron-phonon contribution, omitted in this paper, has now been computed [7] and is not negligible for $\mathrm{ZnO}$ or GaN.

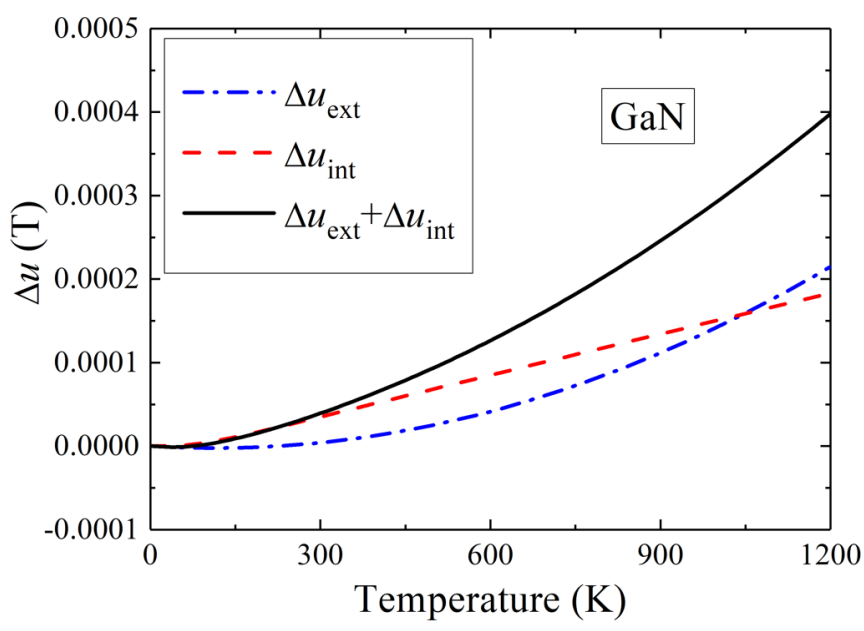

FIG. 2. Thermal shift of the GaN internal parameter $u$. The derivatives $d u / d T$ were given in the original paper as Fig. 1. The notations $\Delta u_{\text {int }}$ and $\Delta u_{\text {ext }}$ are explained in Fig. 1.
[1] H. Iwanaga, A. Kunishige, and S. Takeuchi, J. Mater. Sci. 35, 2451 (2000).

[2] H. Karzel, W. Potzel, M. Köfferlein, W. Schiessl, M. Steiner, U. Hiller, G. M. Kalvius, D. W. Mitchell, T. P. Das, P. Blaha, K. Schwarz, and M. P. Pasternak, Phys. Rev. B 53, 11425 (1996).

[3] E. H. Kisi and M. M. Elcombe, Acta Cryst. C 45, 1867 (1989).
[4] J. Albertsson, S. C. Abrahams, and Å. Kvick, Acta Cryst. B 45, 34 (1989).

[5] J. Liu and P. B. Allen, Internal and external thermal expansions of wurtzite $\mathrm{ZnO}$ from first principles (unpublished).

[6] S. Jachalke, P. Hofmann, G. Leibiger, F. S. Habel, E. Mehner, T. Leisegang, D. C. Meyer, and T. Mikolajick, Appl. Phys. Lett. 109, 142906 (2016).

[7] J. Liu and S. T. Pantelides, Phys. Rev. Lett. 120, 207602 (2018). 